\title{
O CONTEXTO DE TRABALHO E OS FATORES INTERVENIENTES AO CONSUMO DE PESQUISA POR ENFERMEIROS ASSISTENCIAIS*
}

\author{
Ítalo Rodolfo Silva', Thiago Privado da Silva², Maria José Carvalho Ferreira ${ }^{3}$, \\ Sabrina Ayd Pereira José ${ }^{4}$, Joséte Luzia Leite ${ }^{5}$
}

\begin{abstract}
RESUMO: Objetivo: compreender, na perspectiva de enfermeiros assistenciais e pesquisadores, as influências que o contexto de trabalho exerce sobre o consumo de pesquisa por enfermeiros assistenciais. Metodologia: pesquisa qualitativa cujos referenciais teórico e metodológico foram a Teoria da Complexidade e a Teoria Fundamentada nos Dados. Participaram 10 enfermeiros assistenciais e seis enfermeiros pesquisadores, vinculados a instituições públicas da cidade do Rio de Janeiro. Os dados foram captados mediante entrevista semiestruturada, entre outubro de 2014 e março de 2015. Resultados: dimensões da cultura organizacional e do gerenciamento de enfermagem surgiram como aspectos influenciadores do consumo de pesquisa pelo enfermeiro assistencial. Nestes, estão: liderança, recursos humanos, físicos, flexibilidade e incentivo para a busca de competências a partir da pesquisa. Conclusão: na dimensão assistencial, o consumo de pesquisa é influenciado por fatores multifacetados, relacionados com a valorização do capital humano que sofre e exerce influência na dinâmica organizacional a partir do conhecimento científico atualizado. DESCRITORES: Enfermagem; Gestão do conhecimento; Pesquisa; Organização institucional; Cultura organizacional.
\end{abstract}

\section{THE WORK CONTEXT AND THE INTERVENIENT FACTORS FOR THE CONSUMPTION OF RESEARCH BY CLINICAL NURSES}

\begin{abstract}
Objective: to comprehend, from the perspectives of clinical nurses and nurse researchers, the influences that the work context exerts on the consumption of research by clinical nurses. Methodology: qualitative study which considered the Complexity Theory and Data-Based Theory as the theoretical and methodological references. Participants were 10 clinical nurses and six nurse researchers, linked to public institutions of the city of Rio de Janeiro. Data were collected through a semi-structured interview between October 2014 and March 2015. Results: dimensions of the organizational culture and nursing management emerged as influential aspects for the consumption of research by the clinical nurse. These were: leadership, human and physical resources, flexibility and incentive to seek skills from the research. Conclusion: in the care dimension, the consumption of research is influenced by multifaceted factors, related to the valorization of the human capital that suffers and exerts an influence on the organizational dynamics from the updated scientific knowledge.

DESCRIPTORS: Nursing; Knowledge management; Research; Institutional organization; Organizational culture.
\end{abstract}

\section{EL CONTEXTO DE TRABAJO Y LOS FACTORES INTERVINIENTES EN EL CONSUMO DE INVESTIGACIONES POR ENFERMEROS ASISTENCIALES*}

RESUMEN: Objetivo: comprender, en la perspectiva de enfermeros asistenciales e investigadores, las influencias del contexto de trabajo sobre el consumo de investigaciones por enfermeros asistenciales. Metodología: investigación cualitativa utilizándose la Teoría de la Complejidad y la Teoría Fundamentada en los Datos como referenciales teórico y metodológico. Participaron 10 enfermeros asistenciales y seis enfermeros investigadores, vinculados a instituciones públicas de la ciudad de Rio de Janeiro. Los datos fueron recolectados mediante entrevista semiestructurada, entre octubre del 2014 y marzo del 2015. Resultados: dimensiones de la cultura organizacional y de la gestión de enfermería surgieron como aspectos que influyen en el consumo de investigaciones por el enfermero asistencial. Estos abarcan: liderazgo, recursos humanos, físicos, flexibilidad e incentivo a la búsqueda de competencias con base en la investigación. Conclusión: en la dimensión asistencial, factores polifacéticos influyen en el consumo de investigaciones, relacionados a la valoración del capital humano que sufre e influye en la dinámica organizacional a partir del conocimiento científico actualizado.

DESCRIPTORES: Enfermería; Gestión del conocimiento; Investigación; Organización institucional; Cultura organizacional.

\footnotetext{
*Artigo extraído da tese de doutorado intitulada Gestão do conhecimento científico: conexões entre a pesquisa e o gerenciamento do cuidado de enfermagem no contexto da adolescência, vinculada ao Programa de Pós-Graduação da Escola de Enfermagem Anna Nery, da Universidade Federal do Rio de Janeiro, 2015.

${ }^{1}$ Enfermeiro. Doutor em Enfermagem. Professor do Curso de Enfermagem da Universidade Federal do Rio de Janeiro. Professor permanente do Programa de Pós-Graduação da Escola de Enfermagem Anna Nery, da Universidade Federal do Rio de Janeiro. Rio de Janeiro, RJ, Brasil.

${ }^{2}$ Enfermeiro. Doutor em Enfermagem. Professor do Curso de Enfermagem da Universidade Federal do Rio de Janeiro. Macaé, RJ, Brasil.

${ }^{3}$ Enfermeira. Mestre em Enfermagem. Primeiro-Tenente do Corpo de Saúde da Marinha do Brasil. Rio de Janeiro, RJ, Brasil.

${ }^{4}$ Enfermeira. Doutora em Enfermagem. Professora Adjunta do Curso de Enfermagem da Universidade Federal do Rio de Janeiro. Macaé, RJ, Brasil.

${ }^{5}$ Enfermeira. Doutora em Enfermagem. Professora Emérita da Universidade Federal do Estado do Rio de Janeiro.Docente permanente do Programa de Pós-Graduação da Escola de Enfermagem Anna Nery, da Universidade Federal do Rio de Janeiro. Rio de Janeiro, RJ, Brasil.
} 


\section{INTRODUÇÃO}

A cultura organizacional das instituições de trabalho está alicerçada em sistemas de relações, poder e significados estabelecidos entre pessoas e nichos hierárquicos que constituem estruturas formais e informais dessas organizações, incluindo os sistemas promotores e mantenedores de saúde e de cuidados, o que implica considerar a enfermagem como importante elemento desse processo $^{(1-2)}$.

Nessa conjuntura, destaca-se o valor do pensamento complexo ao permitir valorizar a multidimensionalidade da cultura organizacional de uma instituição de saúde, em especial a compreensão sobre a importância das interações estabelecidas entre as pessoas para a construção de um modus operandi dos trabalhadores capaz de sustentar a identidade da própria instituição em que estão inseridos ${ }^{(3)}$. Logo, faz-se necessário considerar que o contexto de trabalho do enfermeiro não se limita ao espaço geográfico ou à filosofia institucional isolada, haja vista o contexto se configurar com o sistema vivo de conexões ${ }^{(4-5)}$.

Sendo sistema, comporta dimensões que projetam singularidades na expressão do todo organizacional, uma vez que este emerge e se mantém a partir das interações entre os elementos que o constituem $^{(3)}$. Dito isso, corrobora-se o princípio da complexidade - o hologramático -, cuja parte está no todo e o todo em cada parte ${ }^{(3)}$.

Destarte, tomando esse princípio como elemento basal, há que se destacar a dinâmica organizacional e o capital humano como polos que se complementam e influenciam o processo de trabalho do enfermeiro $^{(2,6)}$ e, por conseguinte, as práticas científicas que permeiam esse processo. Nesse ínterim, está o consumo de pesquisa como fundamento para a tomada de decisão pautada em conhecimento científico atualizado(7-8).

As influências contextuais podem, dessa forma, exercer impactos nos sistemas de significados e na prática dos enfermeiros assistenciais para o consumo de pesquisa científica ${ }^{(7)}$. Todavia, esses contextos também sofrem influências de outras realidades, sejam elas positivas ou negativas, especialmente dos principais espaços produtores da ciência da enfermagem: as universidades ${ }^{(9)}$.

Logo, os enfermeiros pesquisadores inseridos no cenário acadêmico configuram-se como elementos que podem influenciar o consumo de pesquisa por enfermeiros assistenciais, em seus espaços laborais. Contudo, a literatura aponta a necessidade de estratégias que permitam a convergência entre o desenvolvimento de pesquisa e a dimensão assistencial ${ }^{(7-8)}$. Essa realidade fundamenta a seguinte questão: de que forma os enfermeiros inseridos na assistência e na pesquisa compreendem as implicações do contexto de trabalho para o consumo de pesquisa pelos enfermeiros assistenciais?

A relevância imbuída nessa problemática repousa na importância de se compreender os fatores intervenientes projetados pelo contexto laboral em relação ao consumo de pesquisa pelos enfermeiros inseridos na assistência, tomando como base elucidativa a percepção de quem vivencia essa realidade e de quem poderá influenciá-la ${ }^{(9)}$.

Ademais, as demandas emergentes de saúde e de cuidados consideram a necessidade da sedimentação científica atualizada para melhor atendê-las. Desse modo, foi objetivo da pesquisa compreender, na perspectiva de enfermeiros assistenciais e pesquisadores, as influências que o contexto de trabalho exerce sobre o consumo de pesquisa por enfermeiros assistenciais.

\section{METODOLOGIA}

Estudo explicativo, de abordagem qualitativa, que teve como referenciais teórico e metodológico a Teoria da Complexidade ${ }^{(3)}$ e a Teoria Fundamentada nos Dados (TFD), respectivamente.

A TFD é um método desenvolvido a partir de um conjunto de recursos analíticos que, sistematicamente conduzidos, possibilitam gerar uma matriz teórica explicativa do fenômeno de pesquisa ${ }^{(10)}$. Nesse sentido, favorece a compreensão sobre os fatores que estruturam, condicionam e/ou influenciam um fenômeno. 
Os dados apresentados emergem de dois grupos amostrais, a saber: enfermeiros assistenciais e enfermeiros pesquisadores. Foram critérios de inclusão para o grupo de enfermeiros assistenciais: tempo de experiência profissional, no cenário atual, igual ou superior a um ano; critérios de exclusão: enfermeiro que estivesse cursando pós-graduação na modalidade stricto sensu, haja vista as peculiaridades desse contexto de formação em relação ao consumo e desenvolvimento de pesquisa.

Para o grupo de enfermeiros pesquisadores, foram critérios de inclusão: possuir título de doutor, em qualquer área de conhecimento; estar vinculado a um grupo de pesquisa cadastrado no Conselho Nacional de Desenvolvimento Científico e Tecnológico - CNPq, com linha de pesquisa que se aproximasse do contexto de atuação dos enfermeiros que compuseram o primeiro grupo amostral. Foram excluídos os pesquisadores cuja experiência no gerenciamento de pesquisa estabelecesse tempo inferior a dois anos. Assim, foram selecionados 16 participantes, sendo 10 enfermeiros assistenciais, seis enfermeiros pesquisadores.

Sobre os cenários da pesquisa, faz-se pertinente considerar que, como ciência em construção e prática social, a enfermagem possui distintos espaços de atuação, onde, para cada um deles, poderão existir peculiaridades ao desenvolvimento de pesquisas e para convergência entre resultados científicos e a dimensão assistencial.

A partir desse entendimento, buscou-se um campo de conhecimento e intervenção que necessita ser fortalecido no panorama dos grupos de pesquisa em enfermagem no Brasil. Esse contexto é a adolescência - não como área isolada do saber -, mas como etapa natural do ciclo da vida que requer investimento na formação e desenvolvimento de pesquisas para melhores intervenções pautadas na ciência. Corrobora essa assertiva a escassez de grupos de pesquisa de enfermagem, no Brasil, para essa área específica de conhecimento e atuação, em comparação com as demais fases do ciclo vital ${ }^{(11)}$.

Assim, foram cenários da pesquisa: para o grupo composto por enfermeiros assistenciais, um núcleo de estudos e atenção à saúde do adolescente de um hospital universitário, da capital do Rio de Janeiro. As atividades desenvolvidas nesse núcleo abrangem a assistência à saúde nos níveis de atenção primária, secundária e terciária, estabelecidos pelo Sistema Único de Saúde (SUS). Para os enfermeiros pesquisadores, delimitaram-se, como cenário, grupos de pesquisa cadastrados no Conselho Nacional de Desenvolvimento Científico e Tecnológico(CNPq), vinculados a universidades situadas no Rio de Janeiro.

A seleção dos participantes foi orientada pela amostragem teórica, não probabilística, da TFD, que consiste em maximizar oportunidades comparativas de fatos ou incidentes para determinar como uma categoria varia em termos de suas propriedades e dimensões ${ }^{(10)}$. A coleta de dados foi finalizada ao atingir a saturação teórica, a saber: quando as categorias apresentaram densidade explicativa capaz de contemplar o objeto de pesquisa.

O recrutamento dos enfermeiros assistenciais foi por conveniência; foi utilizada a técnica de bola de neve. Para captar os enfermeiros pesquisadores, realizou-se busca parametrizada na Plataforma Lattes, no campo do Diretório dos Grupos de Pesquisa do CNPq, utilizando-se as estratégias de refinamento descritas no quadro a seguir:

Quadro 1 - Consulta parametrizada para a captação de grupos de pesquisa. Rio de Janeiro, RJ, Brasil, 2017

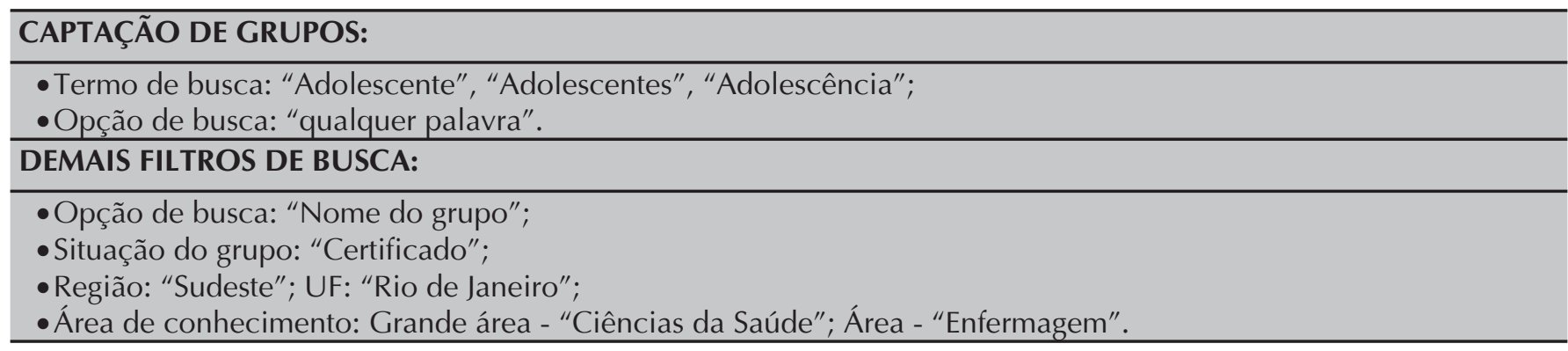

Fonte: Base corrente do Diretório de Grupos de Pesquisa do Conselho Nacional de Desenvolvimento. Científico e Tecnológico - CNPq, Brasil (2015). 
Após selecionar os grupos de pesquisa, cada pesquisador foi convidado a participar do estudo, mediante correspondência eletrônica, pelo e-mail cadastrado no Currículo Lattes.

A entrevista semiestruturada foi empregada como técnica para coleta de dados, realizadas no período de outubro de 2014 a maio de 2015, individualmente, e gravadas em meio digital. A entrevista foi iniciada com a seguinte pergunta indutora: "Fale-me sobre como você percebe a influência do contexto de trabalho para o consumo de pesquisa do enfermeiro que está na assistência". Os locais das entrevistas foram os cenários descritos anteriormente, em ambientes reservados. Para o grupo de enfermeiros pesquisadores, o local de coleta foi a própria instituição de ensino cujo grupo de pesquisa estava vinculado.

A análise dos dados se deu por codificação que, na TFD, consiste em análise comparativa em três níveis: aberta, axial e seletiva ${ }^{(10)}$. Na codificação aberta, os conceitos foram identificados por comparações entre propriedades e dimensões dos dados. Nessa etapa, surgiram os códigos preliminares a partir dos títulos atribuídos para cada incidente, ideia ou evento. Os códigos preliminares foram agrupados em códigos conceituais ${ }^{(10)}$.

Na codificação axial, ocorreu o agrupamento dos códigos conceituais para formar as categorias e subcategorias $^{(10)}$. Nessa etapa, iniciou-se o processo de reagrupamento dos dados que foram separados na codificação aberta, visando uma explicação densa do fenômeno.

A codificação seletiva consistiu na comparação e análise das categorias e subcategorias, processo este realizado de forma contínua que objetiva desenvolver as categorias, integrar e refinar a matriz teórica fazendo emergir o fenômeno central ${ }^{(10)}$.

As categorias foram ordenadas segundo o modelo paradigmático ${ }^{(10)}$, esquema este que possibilita coerência explicativa entre as dimensões que sustentam o objeto de estudo. Sua estrutura se dá a partir dos componentes: fenômeno, condições causais, condições intervenientes, contexto, estratégias de ação/interação e consequências.

A pesquisa foi aprovada, no segundo semestre de 2014, pelos Comitês de Ética em Pesquisa (CEP) da Escola de Enfermagem Anna Nery, da Universidade Federal do Rio de Janeiro, com o parecer de aprovação de no 30438114000005238 e pelo CEP do Hospital Universitário Pedro Ernesto, da Universidade do Estado do Rio de Janeiro, com o parecer de aprovação de no 30438114030015259 . A participação se deu de forma voluntária, após esclarecimento e assinatura do Termo de Consentimento Livre e Esclarecido. Para manter o anonimato dos participantes, os trechos de depoimentos apresentados ao longo do artigo foram designados por acrônimos, de acordo com o grupo amostral e seguidos da sequência de sua respectiva entrevista. Desse modo, o $1^{\circ}$ grupo (EAn⿳o: Enfermeiro Assistencial) e o $2^{\circ}$ grupo (EPno: Enfermeiro Pesquisador).

\section{RESUltados}

Os resultados derivam da tese de doutorado "Gestão do Conhecimento Científico: conexões entre a pesquisa e o gerenciamento do cuidado de enfermagem no contexto da adolescência". Contudo, em decorrência da densidade teórica, esse artigo aborda a categoria "Implicações do contexto de trabalho para o consumo de pesquisa por enfermeiros assistenciais", que no emprego do modelo paradigmático, se configura como condição interveniente às conexões entre pesquisa e assistência. Nessa perspectiva, apresenta-se a primeira subcategoria.

\section{Implicações do contexto de trabalho que (des)favorecem o consumo de pesquisa pelo enfermeiro assistencial.}

O contexto de trabalho apresenta peculiaridades para o incentivo ao consumo de pesquisa, porém, cumpre destacar a natureza contextual de onde emergem as experiências dos enfermeiros assistenciais 
participantes dessa pesquisa, por se tratar de um hospital público universitário, conforme retratado nos trechos a seguir.

Aqui é uma instituição em que os profissionais correm atrás, quando temos alguma dúvida buscamos os manuais do Ministério da Saúde, por exemplo. (EA1)

[...] por termos um contato muito próximo com uma instituição de ensino, noto que há mudança, onde ocorre uma aproximação (pesquisa e prática assistencial), está acontecendo essa troca de informações. (EA6)

Apesar do exposto, aspectos como recursos humanos e sobrecarga de trabalho foram sinalizados como elementos que influenciam a prática de consumo de pesquisa pelos enfermeiros assistenciais. Esses fatores intervenientes são evidenciados pelos participantes do estudo a partir de suas experiências em outros contextos laborais.

Quando se tenta colocar em prática, faltam recursos também. Acho que tem muita coisa envolvida, recursos humanos, materiais [...] em outra instituição eu sou enfermeira para 65 pacientes, ou seja, nem o processo de enfermagem consigo fazer. (EA3)

Em outra realidade que a gente conhece, você vê o profissional sobrecarregado [...] Como profissional, ele se vê desmotivado pelo cansaço físico e mental. (EA5)

Os enfermeiros pesquisadores reconhecem os elementos gerenciais intervenientes à prática de consumo de pesquisa apresentados pelos enfermeiros assistenciais. Assim, reforçam o entendimento sobre as implicações limitadoras das condições de trabalho para a conexão entre o acesso aos resultados de pesquisa e a assistência de enfermagem.

[...] a principal dificuldade é de o enfermeiro assistencial conseguir participar dos grupos de pesquisa, por conta da demanda de trabalho[...] às vezes, tem mais de um emprego. (EP1)

O que se torna mais preocupante é que, às vezes, esse enfermeiro vem para fazer o seu estudo conosco e querem incorporar logo os resultados no serviço e ele percebe que, de imediato, isso não é possível, por conta da infraestrutura, da própria equipe que tem que ser treinada. (EP2)

Além dos recursos físicos e humanos, a dimensão gerencial do serviço de saúde e o gerenciamento em enfermagem foram pontuados como elementos que influenciam a prática de consumo de pesquisa pelo enfermeiro assistencial. A partir dessa conjuntura, emergiu a próxima subcategoria.

\section{Dinâmica gerencial e suas implicações para o consumo de pesquisa na assistência de enfermagem.}

Essa realidade demonstra a influência da gerência/gestor na (des)motivação do enfermeiro assistencial para o consumo de pesquisa.

A gerência pode incentivar motivando esse profissional na busca pelo conhecimento.(EA5)

Na dimensão assistencial, se o gestor não apoiar, você vai ficar sofrendo no seu processo de trabalho sem conseguir mudar [...] às vezes, a pessoa tem o conhecimento, mas não tem o poder conferido pelo cargo de sua posição na instituição para mudar alguma coisa. (EP6)

Como fenômeno complexo, é desejável que a gerência reconheça a motivação para o consumo de pesquisas pelo enfermeiro assistencial em sua dinâmica multifacetada. Nessa perspectiva, surge a flexibilidade no processo de trabalho, a gestão do tempo e o incentivo financeiro como possibilidades estratégicas para conectar resultados de pesquisa e assistência de enfermagem, tendo como base o consumo de conhecimento científico atualizado.

[...] deveria ter um incentivo maior para o enfermeiro estudar, não só o incentivo financeiro, mas, se, de repente, um incentivo mais no trabalho, uma liberação para a pesquisa, que é a realidade daqui, mas nos outros lugares isso não acontece. (EA3)

[...] O profissional sabe que ele não vai usar o tempo dele de folga, o tempo dele de descanso para fazer pesquisa científica, o que, de certa forma, acho correto, porque se ele vai buscar o conhecimento científico para atuar na assistência, por que não utilizar o seu tempo de trabalho? (EA5) 
[...] tem a questão da valorização, que não é só financeira, mas o fator financeiro é importante quando, por exemplo, se tem na instituição o plano de cargos e carreira, isso é um ponto de valorização.(EP5)

O distanciamento entre pesquisa científica e assistência de enfermagem, ainda no campo da gerência, é fortalecido pelo modelo de liderança, bem como pelas estratégias de intervenção utilizadas para a convergência dessas dimensões. Diante disso, a liderança autocrática e o modelo de educação linear são apresentados como fatores contraproducentes às conexões necessárias para o consumo de pesquisa pelo enfermeiro assistencial.

[...] ela [chefe] veio com esse entusiasmo, mas sem o preparo de transição da equipe, ela chegou impondo [...] mas sendo autocrata não dá! Não adianta você querer impor. Você tem que começar mostrando o teu trabalho primeiro [...] fazer as pessoas entenderem o porquê essas mudanças serão importantes [...] É como o modelo de ensino que a gente tem. Na prática é a mesma coisa, onde ele chega dizendo: eu sou o professor, eu tenho o conhecimento e vocês tem que aprender. (EA6)

O importante é mostrar pelo exemplo. Se apenas quiser decretar como deve ser feito, ninguém vai vestir a camisa. Tem que saber como falar, tem que ser líder de verdade. (EA9)

Para os participantes, da convergência entre o gerenciamento de enfermagem e o consumo de resultados de pesquisa pelos enfermeiros resultam impactos positivos para a dinâmica organizacional da instituição e para o processo de trabalho.

[...] esse enfermeiro gestor tem que valorizar o enfermeiro que busca o conhecimento científico e aí traçar uma estratégia para ajudar, porque esse enfermeiro terá um trabalho diferenciado. (EA5)

Orientei uma tese de doutorado sobre o processo de alta da criança com necessidade especial de saúde[...] Se a alta é uma ação administrativa, ela tem que ser uma ação interdisciplinar, aí ela se transformou na coordenadora do processo de alta [...] e está desenvolvendo a coordenação do processo de alta em uma ação interdisciplinar. (EP4)

A dinâmica gerencial corresponde, desse modo, implicações que se manifestam na ordem do poder institucional, permeando elementos como liderança, cultura organizacional e, sobretudo, capacidade da gerência em compreender o consumo de pesquisa pelos enfermeiros assistenciais como possibilidade para o desenvolvimento do capital humano qualificado no atendimento das demandas emergentes de saúde.

\section{- DISCUSSÃO}

A filosofia organizacional de um hospital universitário apresenta fatores que podem influenciar a dinâmica de trabalho do enfermeiro e que, por sua vez, não são generalizáveis a outros cenários laborais, com destaque para as demandas científicas, haja vista a potencial aproximação com práticas de ensino e pesquisa; além da suposta relação de estabilidade empregatícia e as possibilidades de incentivo profissional, mediante o plano de cargos e carreira ${ }^{(12)}$.

Por outra vertente, a prática de consumo de pesquisa pelo enfermeiro assistencial é influenciada pelas condições de trabalho que constituem fatores intervenientes não particularizados ao contexto de um hospital universitário, ao passo que são pontuados pela literatura como situações transversais para diversos cenários em que o enfermeiro está inserido; com ênfase para a variável tempo e a sobrecarga de trabalho gerada pela insuficiência de recursos humanos e materiais; infraestrutura e jornada de trabalho ${ }^{(13-15)}$.

Sendo assim, as conexões entre pesquisa e processo de trabalho da enfermagem, no âmbito assistencial, deparam-se com limitações processuais do contexto laboral, que, de certo modo, colaboram para uma realidade que distancia possibilidades de uma enfermagem autônoma e valorizada, mediante o empoderamento científico necessário para a efetivação de seu processo de trabalho ${ }^{(12,16)}$. Nessa realidade, os desafios permeiam da dificuldade de acesso aos resultados de pesquisa, decorrentes do déficit de recursos materiais/infraestrutura, às possibilidades para a implementação desses conhecimentos na prática. 
Essa situação pode ser explicada pelo pensamento complexo, no princípio da ecologia da ação, em que toda e qualquer ação, na medida em que entra no sistema de interações do cenário em que ela ocorre, escapa, progressivamente, à vontade de seu autor ${ }^{(3)}$. Desse modo, além de correr o risco de fracassar, as ações podem sofrer desvios ou distorções de seu sentido original.

Depreende-se desse fato o entendimento de que, a intenção do enfermeiro assistencial em consumir pesquisa pode não ser suficiente para efetivar suas ações e decisões na busca por subsídios e reorientações de suas práticas de trabalho, uma vez que o efeito da ação não depende, apenas, da intenção de seu autor, mas, também, das condições próprias do contexto em que ela se desenvolve, comportando, desse modo, o princípio da incerteza ${ }^{(3)}$.

Nesse sentido, os dados destacaram a gerência como elemento capaz de subsidiar estratégias para lidar com as incertezas projetadas na ecologia da ação, especialmente no que se refere aos possíveis mecanismos inter-retroativos entre a intenção/necessidade do enfermeiro em consumir pesquisa e a utilização desses conhecimentos em seu processo de trabalho. Para tanto, a gerência assume destaque frente aos mecanismos de incentivo ao desenvolvimento do capital humano institucional, bem como o papel da liderança nesse processo ${ }^{(2,17)}$.

Ademais, a adesão a novos paradigmas ou modelos assistenciais para a reorientação das práticas de trabalho da enfermagem se constitui em fenômeno que apresenta características objetivas e subjetivas, de ordem individual e coletiva, mas, que está diretamente relacionada com o campo de influências contextuais sobre esses trabalhadores. Tais influências podem motivar ou desmotivar o enfermeiro no desempenho de seu trabalho ${ }^{(18)}$.

Com efeito, tomando a liderança pela capacidade de influenciar as pessoas e estimulá-las para o desenvolvimento de um objetivo comum ${ }^{(2,19)}$, os dados da pesquisa sinalizam para o fato de que, no âmbito da gerência, o contexto pode contornar potenciais situações adversas ao consumo de pesquisa pelo enfermeiro assistencial, com vistas ao seu desenvolvimento e, consequentemente, à qualidade da sua assistência.

Para que essas possibilidades sejam convertidas em realidades, torna-se necessário que a gerência valorize os recursos humanos de enfermagem, entendendo que são estes recursos que estruturam a força motriz para a concretização de mudanças no serviço ${ }^{(17)}$, pois, se os desafios que emergem diante das novas demandas de saúde e de cuidados convergem para o desenvolvimento de competências profissionais, e em especial da enfermagem, numa era fundamentada nos princípios da ciência, inovação e tecnologia, faz-se indispensável pensar, propor e implementar estratégias voltadas para a educação permanente do profissional, o que sugere, destarte, a necessidade de maior flexibilidade e visão ampliada sobre os conhecimentos exigidos para a formação e o desempenho profissional ${ }^{(20)}$.

A relação contexto/capital humano estabelece, portanto, tecedura que inviabiliza pensar as conexões entre consumo de pesquisa e processo de trabalho de enfermeiros assistenciais de forma dissociada; com efeito, retoma-se o sentido de inerência pelo qual o consumo de pesquisa só terá sentido ao passo que puder ser traduzido para a realidade. Assim, tornar-se-á possível avançar diante dos desafios da atual conjuntura dos sistemas de saúde em meio às demandas da sociedade do conhecimento ${ }^{(21)}$.

\section{CONCLUSÃO}

Acerca da dinâmica organizacional do serviço de saúde, os resultados sinalizaram a especificidade contextual de onde os enfermeiros assistenciais se expressam como fator que exerce influência na aproximação desses trabalhadores com a pesquisa científica. Entretanto, os dados revelam potencial transversalidade em relação aos fatores intervenientes para a prática de consumo de pesquisa na esfera assistencial, pois esses mesmos enfermeiros relataram que presenciam situações semelhantes em outros contextos de atuação da enfermagem.

Um dos principais fatores intervenientes ao consumo de pesquisa pelos enfermeiros assistenciais, na percepção dos participantes, está na gerência e seus desdobramentos em relação ao desenvolvimento de capital humano. Nesse processo, abrange desde mecanismos institucionais que permitam flexibilidade 
ao trabalhador para aprimorar suas competências para a pesquisa, como incentivo à participação em eventos e/ou capacitações, plano de cargos e carreira, bem com o próprio processo de liderança, em que pese a capacidade de motivar o profissional para o consumo de ciência atualizada. Entretanto, os recursos físicos e humanos também exercem influência nesse processo, de modo a inviabilizar ou dificultar, por exemplo, o acesso remoto às informações de pesquisas atuais, ou mesmo a implementação de práticas a partir do consumo destas.

Os dados não revelaram especificidade em relação ao contexto de conhecimento e atuação da adolescência. Diante disso, sinalizam para a possibilidade de transversalidade dessa problemática para além de uma área isolada, conforme destacado ao longo do artigo. Todavia, por se tratar de um fenômeno multifacetado, é recomendável que estudos similares sejam desenvolvidos em cenários distintos de atuação, bem como em participantes diversos, dentre os quais os gestores dos serviços de saúde e de enfermagem. Cumpre destacar que a ausência de dados desses atores implica em uma potencial limitação da pesquisa em pauta.

\section{- REFERÊNCIAS}

1. Silva TP, Silva MM, Alcantara LM, Silva IR, Leite JL. Establishing action/interaction strategies for care delivery to hospitalized children with chronic conditions. Esc Anna Nery. [Internet] 2015;19(2) [acesso 23 nov 2016]. Disponível: http://dx.doi.org/10.5935/1414-8145.20150037.

2. Frdique MJ, Mendes L. Efeitos da liderança na melhoria da qualidade dos cuidados de enfermagem. Rev Enf Ref. [Internet]2013;3(10) [acesso em 16 set 2015]. Disponível: http://www.scielo.mec.pt/scielo.php?script=sci_ arttext\&pid=S0874-02832013000200006.

3. Morin E. Ciência com consciência. 13aㅗ ed. Rio de Janeiro: Betrand; 2010.

4. Pivoto FL, Lunardi Filho WD, Lunardi VL, Silva PA. Organization of work and the production of subjectivity of the nurse related to the nursing process. Esc Anna Nery. [Internet] 2017;21(1) [acesso em 18 fev 2017]. Disponível: http:// dx.doi.org/10.5935/1414-8145.20170014.

5. Felli VEA, Peduzzi M. O trabalho gerencial em enfermagem. In: Kurcgant P, organizadora. Gerenciamento em Enfermagem. 2a edição. Rio de Janeiro: Guanabara Koogan; 2014.

6.dos Santos JLG, Lima MADS, Pestana AL, Colomé ICS, Erdmann AL. Strategies used by nurses to promote teamwork in an emergency room. Rev Gaucha Enferm. [Internet] 2016;37(1) [acesso em 27 jan 2017]. Disponível: http://dx.doi. org/10.1590/1983-1447.2016.01.50178.

7. Dalheim A, Harthug S, Nilsen RM, Nortvedt MW. Factors influencing the development of evidence-based practice among nurses: a self-report survey. BMC Health Serv-Res. [Internet]2012;12(362) [acesso em 25 maio 2017]. Disponível: https://doi.org/10.1186/1472-6963-12-367.

8. Ferreira MA. O clássico e o emergente: desafios da produção, da divulgação e da utilização do conhecimento da enfermagem. Rev bras enferm. [Internet] 2013;66(n.esp) [acesso em 20 nov 2016]. Disponível: http://dx.doi.org/10.1590/ S0034-71672013000700006.

9. Valença CN, Santos RCA, Medeiros SM, Guimarães J, Germano RM, de Miranda FAN. Reflexões sobre a articulação entre o homo faber e o homo sapiens na enfermagem. Esc Anna Nery. [Internet] 2013;17(3) [acesso em 16 dec 2016]. Disponível: http://dx.doi.org/10.1590/S1414-81452013000300023.

10. Strauss AL, Corbin J.Pesquisa qualitativa: técnicas e procedimentos para o desenvolvimento de teoria fundamentada. $2^{\underline{a}}$ ed. Porto Alegre: Artmed; 2008.

11. Christoffel MM, de Souza TV, de Silveira ACD, Valente EV, Meireles JR, Silva PL. Grupos de pesquisas em enfermagem na área do recém-nascido, da criança e do adolescente: perfil e tendência. Texto Contexto Enferm. [Internet] 2011; 20(n.esp) [acesso 12 mai 2015]. Disponível: http://www.scielo.br/pdf/tce/v20nspe/v20nspea19.pdf. 
12. Costa FG, Veghetti HH, Matinello DFG, Mendes DP, Terra AC, Alvarez SQ, Lemos LA P. Tendências empreendedoras dos enfermeiros de um hospital universitário. Rev Gaúcha Enferm. [Internet] 2013;34(3) [acesso em 18 dez2016]. Disponível: http://dx.doi.org/10.1590/S1983-14472013000300019.

13. Schmaeller R, Gelbcke FL. Indicators for the measurement of emergency nursing personnel. Texto context - enferm. [Internet] 2013;22(4) [acesso em 16 dez 2016].Disponível:http://dx.doi.org/10.1590/S0104-07072013000400013.

14. Lorenzetti J, Oro J, Matos E,Gelbke FL. Work organization in hospital nursing: literature review aproach.Texto Contexto Enferm. [Internet] 2014;23(4) [acesso em 16 dez 2016]. Disponível: http://dx.doi.org/10.1590/010407072014001510012.

15.Bogossian F, Winters-chang P, Tuckett HA. "Thepure hard slong that nursing is...": a qualitative analysis of nursing work. J. Nus. Scholarsh. [Internet] 2014;46(4) [acesso em 25 ago 2017]. Disponível: https://doi.org/10.1111/jnu.12090.

16. Oelke ND, Lima MADS, Acosta AM. Knowledge translation: translating research into policy and practice. Rev Gaucha Enferm. [Internet] 2015;36(3) [acesso em 18 mai 2017]. Disponível: http://dx.doi.org/10.1590/19831447.2015.03.55036.

17. Camelo SHH, Rocha FLR, Chaves LDP, Silva VLS, Soares MI. Competências profissionais e estratégias organizacionais de gerentes de enfermagem. Cienc. enferm. [Internet] 2016;22(1) [acesso em 22 jan 2017]. Disponível: http://dx.doi. org/10.4067/S0717-95532016000100007.

18. da Silva LJ, Leite JL,da Silva TP, da Silva LR, Scochi CG. Nurses aderence to the Kangoroo Care Method: support for nursing care management. Rev Latino-Am Enfermagem. [Internet] 2015;23(3) [acesso em 10 jan 2017]. Disponível: http://dx.doi.org/10.1590/0104-1169.0339.2579.

19. Nunes EMGT, Gaspar MFM. Leadership in nursing and patient satisfaction in hospital context. Rev. Gaúcha Enferm. [Internet] 2016;37(2) [acesso em 22 jan 2017].Disponível: http://dx.doi.org/10.1590/1983-1447.2016.02.55726.

20. Amestoy SC, Backes VMS, Thofehm MB, Martini JG, Meirelles BHS, Trintade LL. Conflict management: challenges experienced by nurse-leaders in the hospital environment. Rev Gaúcha Enferm. [Internet] 2014;35(2) [acesso em 18 nov 2016] .Disponível: http://dx.doi.org/10.1590/1983-1447.2014.02.40155.

21. López-Montesinos MJ,Maciá-Soler L. Doctorate nursing degree in Spain. Rev. Latino-Am Enfermagem. [Internet] 2015;23(3) [acesso em 8 set 2015]. Disponível: http://dx.doi.org/10.1590/0104-1169.0512.2567. 